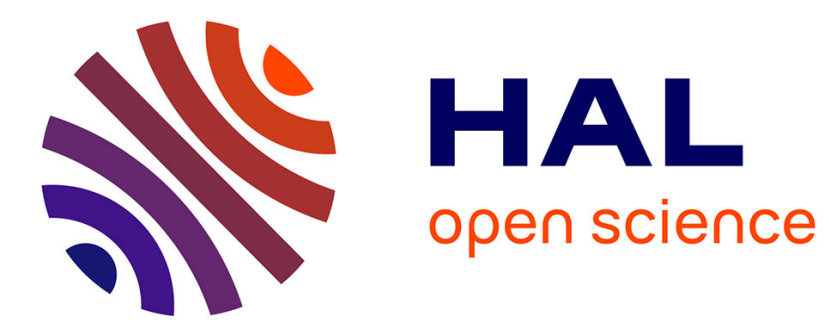

\title{
Water and ions in clays: Unraveling the interlayer/micropore exchange using molecular dynamics
}

Benjamin Rotenberg, Virginie Marry, Rodolphe Vuilleumier, Natalie Malikova, Christian M. Simon, Pierre Turq

\section{- To cite this version:}

Benjamin Rotenberg, Virginie Marry, Rodolphe Vuilleumier, Natalie Malikova, Christian M. Simon, et al.. Water and ions in clays: Unraveling the interlayer/micropore exchange using molecular dynamics. Geochimica et Cosmochimica Acta, 2007, 71, pp.5089-5101. 10.1016/j.gca.2007.08.018 . hal-00369627

\section{HAL Id: hal-00369627 \\ https://hal.science/hal-00369627}

Submitted on 9 Nov 2018

HAL is a multi-disciplinary open access archive for the deposit and dissemination of scientific research documents, whether they are published or not. The documents may come from teaching and research institutions in France or abroad, or from public or private research centers.
L'archive ouverte pluridisciplinaire HAL, est destinée au dépôt et à la diffusion de documents scientifiques de niveau recherche, publiés ou non, émanant des établissements d'enseignement et de recherche français ou étrangers, des laboratoires publics ou privés. 


\title{
Water and ions in clays: Unraveling the interlayer/micropore exchange using molecular dynamics.
}

\author{
Benjamin Rotenberg ${ }^{1,2, *}$, Virginie Marry ${ }^{1}$, Rodolphe Vuilleumier ${ }^{3}$, \\ Natalie Malikova ${ }^{4}$, Christian Simon $^{1}$ and Pierre Turq ${ }^{1}$
}

* e-mail: rotenber@ccr.jussieu.fr

tel:+33 (0)1 442731 18, fax:+33 (0)1 44273228

${ }^{1}$ LI2C, Université Pierre et Marie Curie-Paris6, CNRS, UMR 7612, 4 pl. Jussieu, Paris F-75005, France

${ }^{2}$ ANDRA, Parc de la Croix Blanche, 1/7 rue Jean Monnet, Châtenay Malabry F-92298, France

3 LPTMC, Université Pierre et Marie Curie-Paris6, CNRS, UMR 7600, 4 pl. Jussieu, Paris F-75005, France

${ }^{4}$ LLB, CEA, Gif-sur-Yvette F-91191, France

\begin{abstract}
We present the first microscopic description of the exchange of water and ions between clay interlayers and microporosity. A force field based on Ab-Initio calculations is developed and used in classical Molecular Dynamics simulations. The latter allow to compute the Potential of Mean Force for the interlayer/micropore exchange for water, $\mathrm{Na}^{+}$ and $\mathrm{Cs}^{+}$cations, and $\mathrm{Cl}^{-}$anions. For the simulated water content (water bilayer, with interlayer spacing $15.4 \AA$ ) and salt concentration in the micropore $\left(0.52 \mathrm{~mol} . \mathrm{dm}^{-3}\right)$ the exchange is found to be almost not activated for water and cations, whereas the entrance of an anion into the interlayer is strongly unfavorable $\left(\Delta \mathcal{F} \sim 9 k_{B} T\right)$. Calculations of the diffusion tensor in the interlayer and in the micropore complete the study of the exchange dynamics.
\end{abstract}

Keywords: clay, ionic exchange, anion exclusion, edge, kinetics, molecular dynamics, potential of mean force, diffusion tensor 


\section{Introduction}

Clays consist of negatively charged lamellae, compensated by (solvated) cations which can move in the interlayer spacings originating from the stacking of the mineral layers. These stacks form particles, which also aggregate to form larger structures with a corresponding porosity. Elucidating the transport mechanisms of water and ions through clays is particularly important, since clays are considered as suitable materials for the geological storage of toxic and radioactive waste [Synthèse, 2005, Bradbury and Baeyens, 2003]. The transport of a tracer through a clay sample is averaged over the whole porosity: interlayer, micropores $(\geq 2-3 \mathrm{~nm})$, macropores $(\geq 100 \mathrm{~nm})$ [Bourg et al., 2006]. However a simple average assumes that the tracer can exchange easily between all types of porosities. To interpret the variations of the effective diffusion coefficient $D_{e}$ with the tracer's charge, it is often argued that the porosity accessible to each species depends on its charge and size [Synthèse, 2005, Kato et al., 1995]. Due to the negative charge of clay layers, anions should be repelled by the external surfaces, and excluded from the interlayers. On the contrary, cations are attracted by the surfaces, and may exchange with the natural interlayer counterions. Ions can also be trapped on clay edge sites. Experimental evidence of anionic exclusion and cation exchange are generally indirect: inferred from macroscopic tracer diffusion, or batch measurement of selectivity constants (for exchange) [Synthèse, 2005, Bradbury and Baeyens, 2003, Kato et al., 1995].

Molecular simulations have provided microscopic interpretations of experimental data, related to structural [Skipper et al., 1989, Delville, 1992, 1993, Skipper et al., 1995a,b, Chang et al., 1995, 1997, 1998, Smith, 1998, Sposito et al., 1999, Park and Sposito, 2000, Ferrage et al., 2005a], thermodynamical [Boek et al., 1995a,b, de Siqueira et al., 1997, Shroll and Smith, 1999, Young and Smith, 2000, Whitley and Smith, 2004, Smith et al., 2006, Liu and Lu, 2006, Hensen et al., 2001, Hensen and Smit, 2002, Tambach et al., 2004a,b, 2006] and dynamical [Sutton and Sposito, 2001, Titiloye and Skipper, 2001, Marry et al., 2002, Marry and Turq, 2003, Malikova et al., 2004a,b, 2005, 2006] properties of clay interlayers. The microscopic structure of the water/mineral interface [Wang et al., 2004, Kirkpatrick et al., 2005, Wang et al., 2005, 2006], and adsorption onto clay basal surface [Greathouse and Cygan, 2005, 2006] were also investigated. In a recent paper, Teppen et al. have used microscopic simulations to gain insight into the mechanism of ionic exchange [Teppen and Miller, 2006]. They demonstrated that the thermodynamic driving force of $\mathrm{K}^{+}$to $\mathrm{Cs}^{+}$exchange in montmorillonite is not related to any "selection" of $\mathrm{Cs}^{+}$by the interlayer but rather dominated by the free energy change in the aqueous phase. The ionic exchange reaction between a natural counterion (e.g. $\mathrm{Na}^{+}$) and an ion in solution (possibly a radioactive ion, e.g. ${ }^{137} \mathrm{Cs}^{+}$), can be summarized as:

$$
\mathrm{Cs}_{a q}^{+}+\text {Clay }-\mathrm{Na}^{+} \longrightarrow \mathrm{Na}_{a q}^{+}+\text {Clay }-\mathrm{Cs}^{+}
$$

From a thermodynamical point of view, the study of ionic exchange requires only to consider the initial and final states. However, nothing can be learned on the ionic exchange process and its kinetics without considering explicitely the transition zone between the two states (interlayer and external porosity), which occurs at the clay edges. Indeed, an ion or a water molecule leaving the interlayer (where it diffuses in two dimensions along the clay sheets) for the micropore (where it diffuses in 3D) will experience the 
effect of the interface (close to the edges), and will cross it with a certain probability. This situation is depicted in figure 1. The overall $\mathrm{Na}^{+}$to $\mathrm{Cs}^{+}$ionic exchange is thus a twofold process: (1) $\mathrm{Na}^{+}$release into the micropore and (2) $\mathrm{Cs}^{+}$entrance into the interlayer. Both processes may or may not occur simultaneously, and might be later coupled to a change in interlayer water content, as the $\mathrm{Cs} / \mathrm{Na}$ ratio evolves. Indeed, hydrated homoionic Cs-montmorillonite only exists in the monohydrated state, whereas Na-montmorillonite also exists in the bihydrated (even trihydrated) state. The purpose of the present study is to give microscopic insights into the ionic exchange and the anion exclusion processes, using Molecular Dynamics (MD) simulations. We will consider the interlayer/micropore exchange of each species separately, and focus on the early stages of the overall exchange (when the $\mathrm{Cs} / \mathrm{Na}$ ratio is small, which is the relevant situation for radioactive contaminant transport), so that the interlayer water content corresponds to that of a Na-montmorillonite.

Clarifying the transition between micropore and interlayer is very important to understand the respective contributions of each type of porosity to the overall water and ionic transport. Since no direct experimental data is available on this process on the microscopic scale, microscopic simulations are a reasonable alternative to study it. Such a study remains however a challenge, because it requires to include explicitely in the same simulation box both a clay particle (including interlayers and counterions) and a micropore (containing a bulk electrolyte solution), and a sound description of the particle edges must be adopted.

Clay edges have first been investigated using ab-initio simulations, with the focus on structure and acidity of edges sites [Bickmore et al., 2003, Churakov, 2006]. Indeed, the interpretation of titration curves generally relies on a large number of fitting parameters [Tournassat et al., 2004], partially supported by semi-empirical approches like MUSIC [Hiemstra et al., 1996]. Prediction of acidity constants from first principles aims at supporting the interpretation of these data, which are of great practical importance since they influence dramatically the retention of ions by clays. Such studies have been initiated on short pyrophyllite fragments in vaccuum, and allowed to make some predictions on the relative acidity of edge sites [Churakov, 2006]. More recently, the study of a confined water film on clay edges allowed to observe the proton exchange between acidic edge sites and water adsorbed on the surface [Churakov, 2007]. The system required to study the interlayer/micropore exchange is at present completely out of reach of ab-initio simulations, for two reasons: The size of the system is too large (it involves too many atoms, see below), and the relevant timescale is too long (several nanoseconds). Therefore a simpler description should be adopted. To that end, we first developped a classical force field to describe the edges, based on density functional theory (DFT) calculations on a smaller system. This force field was then used to simulate a larger system containing both interlayer and microporosity, for long times (several nanoseconds).

The microscopic description of the system is not the only challenge. Indeed, the relevant framework to study the exchange kinetics needs to be carefully defined. The standard approach to describe a transition between two states is to evaluate the free energy profile along a reaction coordinate which characterizes the evolution of the process. For a chemical reaction a typical situation is the one illustrated in figure 2: It will occur if the system overcomes a free energy barrier $\Delta \mathcal{F}_{1 \rightarrow 2}^{\#}$; the reverse reaction needs to overcome 
$\Delta \mathcal{F}_{2 \rightarrow 1}^{\#}$, and the overall free energy change is $\Delta \mathcal{F}_{1 \rightarrow 2}=\Delta \mathcal{F}_{1 \rightarrow 2}^{\#}-\Delta \mathcal{F}_{2 \rightarrow 1}^{\#}$. When considering the transition between interlayer and micropore, several questions arise. (1) What is the relevant reaction coordinate to describe the exchange, and what is the shape of the free energy profile, depending on the considered molecule/ion? In particular, the presence of activation barriers $\left(\Delta \mathcal{F}_{1 \rightarrow 2}^{\#}\right.$ or $\left.\Delta \mathcal{F}_{2 \rightarrow 1}^{\#}\right)$ is not a priori obvious. (2) How to describe the dynamics along this free energy profile? This implies the determination of the diffusion coefficient in different regions of the system (interlayer and micropore). We will show that it is in itself a difficult point to address, for the diffusion tensor is anisotropic (2D in the interlayer, 3D in the pore) and depends on the position.

We report here the determination of the free energy profile and the diffusion coefficients along a selected reaction coordinate for the process of water and ionic exchange, using molecular dynamics simulations. Section 2 introduces the system used to investigate the interlayer/micropore exchange, and presents the simulation details (methods and force field). Section 3 discusses the choice of the reaction coordinate, explains how to obtain the free energy profile, and gives the results for water and ionic exchange. Section 4 deals with the evaluation of diffusion coefficients in the interlayer and the micropore. Finally, Section 5 summarizes the major results.

\section{System and simulation method}

\subsection{System}

We simulated a system containing a short clay particle facing a bulk electrolyte solution (see figure 3). The clay is a model montmorillonite with a unit cell of composition $\mathrm{Na}_{0.75} \mathrm{Si}_{8}\left(\mathrm{Al}_{3.25} \mathrm{Mg}_{0.75}\right) \mathrm{O}_{20}(\mathrm{OH})_{4}$ (with dimensions $\left.8.97 \times 5.18 \AA^{2}\right)$. Each layer consists of $2 \times 4$ bulk unit cells (the longest dimension is along $x$ ) and 4 "half-unit cells" on each side (along $y$ ) corresponding to [010] edges and whose structure, illustrated in figure 4, was obtained by cutting the unit cell and saturating the broken bonds with $\mathrm{H}$ or $\mathrm{OH}$ groups.

The interlayer distance (between the layers midplanes) was fixed to $d=15.4 \AA$ which corresponds to a water bilayer [Marry and Turq, 2003]. The micropore has a width of $30 \AA$, divided in $15 \AA$ on each side of the particle. The overall dimensions of the simulation box are $63.0 \times 20.72 \times 30.8 \AA^{3}$, and periodic boundary conditions in all directions are used. Thus the simulated system consists of an infinite stack (along $z$ ) of sheets which are infinite along $y$ and finite along $x$. The simulation box contains 898 water molecules (see Section 2.3). In addition to the interlayer counterions, $6 \mathrm{Na}^{+}$and $6 \mathrm{Cl}^{-}$ions were introduced in the micropore. This corresponds to an electrolyte concentration of $0.52 \mathrm{~mol} . \mathrm{dm}^{-3}$, which is slightly higher than a typical pore ionic strength (closer to $0.1 \mathrm{~mol} . \mathrm{dm}^{-3}$ in the CallovoOxfordian formation, considered for a nuclear waste repository in France). This relatively high concentration has several advantages: Higher statistical accuracy on the ions with less water molecules (this diminishes the computational cost), higher probability for exchange events to occur, and stronger screening of electrostatic interactions between both sides of the clay particle across the pore. 


\subsection{Force field}

The interactions between all atoms were modelled using pairwise potentials:

$$
V_{i j}=\frac{q_{i} q_{j}}{4 \pi \epsilon_{0} r_{i j}}+4 \epsilon_{i j}\left[\left(\frac{\sigma_{i j}}{r_{i j}}\right)^{12}-\left(\frac{\sigma_{i j}}{r_{i j}}\right)^{6}\right]
$$

with $q_{i}$ the (partial) charge of atom $i$, and the Lennard-Jones (LJ) parameters $\epsilon_{i j}$ and $\sigma_{i j}$ are calculated from individual $\epsilon_{i i}$ and $\sigma_{i i}$ using Lorentz-Berthelot mixing rules [Frenkel and Smit, 2002]. This simple force field is completely determined by the atomic parameters $\left\{q_{i}, \epsilon_{i i}, \sigma_{i i}\right\}$. It requires to keep the clay layers rigid whereas more elaborate ones, such as CLAYFF [Cygan et al., 2004], do not suffer from this restriction. Since the purpose of the present study is to develop the theoretical framework to understand the exchange kinetics between interlayer and micropore, and in absence of experimental data to assess the superiority of either force field in presence of the edges, we preferred the use of the simpler model. However the present analysis could be directly reproduced using another

force field. Keeping the $\mathrm{OH}$ bonds orientation fixed in the classical simulation is thus only a first approximation and in further studies we plan to leave these bonds free to rotate.

Water was described with the rigid SPC/E model [Berendsen et al., 1987]. The ions LJ parameters are taken from [Koneshan et al., 1998] and were shown to correctly describe the dynamics of ions in bulk water. Parameters for bulk clay atoms are taken from Smith [Smith, 1998]. The conjunction of all these parameters was shown to reasonably describe the dynamics of water and ions in clay interlayers [Marry and Turq, 2003, Malikova et al., 2005, 2006]. The LJ parameters for edge oxygen atoms were identical to those of the bulk surface oxygen atoms (which are the same as SPC/E water oxygen atoms in the Smith force field).

The partial charges of the edge atoms were then obtained from a fit of the electrostatic potential around the clay fragment. To this aim, Density Functional Theory (DFT) calculations were performed on a short fragment of pyrophyllite (neutral analogue of montmorillonite) terminated at both ends and with the resulting broken bounds saturated with $\mathrm{H}$ atoms or $\mathrm{OH}$ groups. The latter come from the dissociation of water molecules to complete the coordination of edge $\mathrm{Al}$ and $\mathrm{Si}$ atoms, as explained in [Churakov, 2006]. We have used a gradient corrected approximate exchange-correlation functional, BLYP [Becke, 1988, Lee et al., 1988], and a plane wave basis-set with a cut-off at 70 Ry. Norm-conserving pseudo-potentials were then used to represent the core electrons and only valence electrons were explicitly treated. We have used Trouiller-Martins [Troullier and Martins, 1991] pseudo-potentials for H, O and Si and a Bachelet-Hamann-Schlutter [Bachelet et al., 1982] pseudo-potential for $\mathrm{Al}$, in the Kleinman-Bylander representation [Kleinman and Bylander, 1982]. The box was $5.18 \AA$ large, corresponding to the periodicity of the clay and $25 \AA$ in the two non-periodic directions of the clay. Two types of boundary conditions were used for this box: periodic in all directions or only in one direction using the method proposed by Martyna et al. to screen the electrostratic potential [Martyna and Tuckerman, 1999]; results were basically unchanged when comparing both calculations. All DFT calculations reported here were performed with the CPMD package [CPM, 1997-2004].

The charges of the edge atoms were estimated using the Restrained Electrostatic Potential (RESP) method [Laio et al., 2002, Kirchner and Hutter, 2004]. The charges 
of atoms not belonging to the edges (i.e. bulk clay atoms) were kept fixed to the ones of the Smith force field, while we have fitted the charges of the edge atoms to best reproduce the electrostatic potential outside the clay with a restrain keeping the charges close to initial guess values $q_{I}^{*}$. The latter were chosen as the charges of the corresponding bulk clay atoms, so that the resulting partial charges correspond to the rearrangement of the charge density after cutting the edge (and dissociating enough water molecules to complete the coordination of edge $\mathrm{Al}$ and $\mathrm{Si}$ atoms, as explained above). In practice, one first computes the electrostatic potential from the electronic density (and the positions of the atomic nuclei) on a grid, resulting in a set $\left\{V\left(\mathbf{r}_{\mathbf{i}}\right)\right\}_{i=\text { node }}$. The partial charges $\left\{q_{I}\right\}_{I=\text { atom }}$ are then determined by minimizing:

$$
\min _{\left\{q_{I}\right\}}\left[\sum_{i=\text { node }}\left(V\left(\mathbf{r}_{\mathbf{i}}\right)-\sum_{I=\text { atom }} \frac{q_{I}}{4 \pi \epsilon_{0}} \frac{1}{\left\|\mathbf{r}_{\mathbf{i}}-\mathbf{r}_{\mathbf{I}}\right\|}\right)^{2}+\lambda \sum_{I=\text { atom }}\left(q_{I}-q_{I}^{*}\right)^{2}\right]
$$

with $\lambda$ the restrain parameter. This restrain is necessary as the Electrostatic Potential (ESP) method is not well conditioned for an extended system. The strength of the restrain was chosen such that all oxygen atoms have a negative charge, and all other atoms a positive charge as is chemically expected. On a relatively large range of restrain strength, the charges are nearly unchanged, varying only by a few percents (we have used $\lambda=2)$.

The chosen guess charges and restrain strength, while allowing for the RESP procedure to converge, do not influence dramatically the outcome of the equilibration procedure. Indeed, the latter results in differences between initial and final charges of as high as $20 \%$ for $\mathrm{Al}$ atoms and $35 \%$ for Si atoms. Charges on both edges were similar although as mentioned above the two edges are not perfectly symmetric and an average value was used for use in the classical simulation. Both the geometry and the charges were checked employing a cell twice as large, resulting in very similar charges as with the small cell. The atomic coordinates and partial charges obtained from this charge fitting procedure are given in electronic annexes EA-1 and EA-2. Churakov recently demonstrated that the structure predicted by ab-initio simulation in vaccuum of this particular [010] edge was in good agreement with the structure determined in presence of a water film at the edge surface [Churakov, 2007]. This supports the use of the structure and partial charges determined in vaccuum for classical simulation in presence of water.

\subsection{Simulation details}

Water molecules and ions were introduced randomly in the simulation box, and the system was first equilibrated using Monte-Carlo (MC) simulations in the NVT ensemble, for $5.10^{6}$ steps at $1000 \mathrm{~K}$, then $5.10^{6}$ steps at $500 \mathrm{~K}$ and finally $15.10^{6}$ steps at $298 \mathrm{~K}$. The $6 \mathrm{Na}^{+}$ions in the micropore after equilibration were then replaced by $6 \mathrm{Cs}^{+}$, to investigate the $\mathrm{Na}^{+}$to $\mathrm{Cs}^{+}$exchange. After another MC $10^{6}$ steps at $298 \mathrm{~K}$, Molecular Dynamics simulations in the NVT ensemble were performed using the DLPOLY simulation package. The choice of fixed volume is the easiest way to account for the coexistence of a bulk solution (micropore) with a bihydrated clay which occurs experimentally in underground conditions. During equilibration, the water molecules in the simulation box divide themselves between the 
micropore and the interlayer. This results in a water density in the center of the micropore equal to that of bulk water, and 12-13 water molecules per counterion in the interlayer. These results indicate that the chosen number of water molecules is consistent with the coexistence of a bulk micropore and a water bilayer, since the experimental equilibrium distance of $15.4 \AA$ [Calvet, 1973, Fu et al., 1990] was obtained in Monte-Carlo simulations of bulk clays for a similar water content [Marry and Turq, 2003].

The temperature was maintained at $298 \mathrm{~K}$ using a Nosé-Hoover thermostat [Frenkel and Smit, 2002], and the equations of motion for the rigid water molecules were integrated using the SHAKE algorithm. All molecular dynamics simulations were performed with a 1 fs timestep. Ewald summation was used to compute the electrostatic interactions. After MC equilibration, the system was further equilibrated with MD for 500 ps, before a production run of 20 ns.

\section{$3 \quad$ Free energy profile}

\subsection{Reaction coordinate}

As mentioned in the introduction, when considering the interlayer/micropore exchange, one first needs to carefully define what the initial and final states are, and to make an appropriate choice of a reaction coordinate $(\mathrm{RC})$. The abscissa $x$ is a natural order parameter to distinguish the interlayer (initial state characterized by a coordinate $|x| \leq x_{l}$, with $x=0$ at the center of the simulation box) from the micropore (final state, $|x| \geq x_{l}$ ). The frontier $x_{l}$ corresponds to the location of the large variations of the free energy.

In the following, we will argue that $x$ can be in fact chosen as the reaction coordinate. Before doing so, we would like to clarify the meaning of the free energy profile introduced in section 1. This curve represents the free energy along the Minimum Free Energy Path (MFEP) which connects one state point, corresponding to a given molecule/ion in the interlayer, to another (same molecule/ion in the micropore). This is the path in phase-space that the system will follow with maximum likelihood, and it is therefore representative of the exchange process, since the free energy $\mathcal{F}$ of a state is related to its probability of occuring $P$ :

$$
\mathcal{F}=-k_{B} T \ln P+\mathcal{F}_{0}
$$

where $k_{B}$ is Boltzmann's constant, $T$ the temperature, and $\mathcal{F}_{0}$ is a constant ensuring the normalization of $P$. The reaction coordinate is then a curvilinear coordinate along the MFEP. The free energy along the MFEP is referred to as "Potential of Mean Force", because the thermodynamic driving force is the gradient of the free energy profile, which follows the MFEP. Determining the MFEP (and the RC) for a complex system is a formidable task. Only a few methods do not rely on an a priori choice of the reaction coordinate: This is for example the case of the String Method [Maragliano et al., 2006] and Metadynamics [Laio and Parrinello, 2002]. Even in these cases, one assumes that it is a function of some predetermined variables (e.g. distances or angles). Under the assumption that the MFEP can be expressed as very few variables only, one can map the free energy landscape in these variables and determine the MFEP a posteriori. In any case, the strongest assumption lies in the choice of the considered variables. 
A thorough analysis of the choice of $x$ only as the reaction coordinate for the exchange is out of the scope of this paper. We only indicate here why the $z$ coordinate does not seem to matter for this process (an even simpler discussion can be done for the $y$ coordinate). Let us consider the transition of a water molecule between the interlayer and the micropore. The free energy landscape as a function of position can be obtained from Equation 4, since the probability $P$ is simply proportional to the density $\rho(x, z)=$ $\int \rho(x, y, z) \mathrm{d} y$. We thus have $\mathcal{F}(x, z)=-k_{B} T \ln \rho(x, z)+\mathcal{F}_{0}^{\prime}$, with $\mathcal{F}_{0}^{\prime}$ is an arbitrary constant. This formula is of practical use only if the density can be obtained with sufficient statistical accuracy, and we will see in section 3.3 how to treat the problem when this is not the case.

Figure 5 shows the two-dimensional density $\rho_{\mathrm{OW}}(x, z)=\int \rho_{\mathrm{OW}}(x, y, z) \mathrm{d} y$ of water oxygen atoms, with $\rho_{\mathrm{OW}}(x, y, z)$ the usual $3 \mathrm{D}$-density. It is obvious from this figure that the density is almost independent of $z$ in the micropore, whereas in the particle illustrated in figure 1 (corresponding to $|x|<x_{l}$ ) it is non zero only in the interlayer (it strongly depends on $z$ ). Therefore the two-dimensional free energy landscape consists of two mountains (clay sheets) separating two deep canyons (interlayers), the latter being connected to a flat plain (micropore). A molecule forced into the clay particle will thus spontaneously find the interlayer, even if it was not initially facing it (see figure 5). Hence the variations of free energy along the MFEP will only depend on $x$, and we can restrict ourselves to the study of the Potential of Mean Force as a function of $x$ only. This simplistic argument underlines that this choice corresponds more precisely to a particle/micropore exchange, although in practice the exchange is indeed between interlayer (defined not only by the $x$ coordinate, but also by some values of the $z$ coordinate) and micropore. Note that more subtles quantities, such as the coordination number of the transiting species, are not excluded by the above discussion.

\subsection{Density profiles}

The position of the frontier defining the two states, can be estimated through the study of the density profiles of each species. All density profiles clearly reveal, as expected, three distinct regions: the interlayer (approximately for $|x|<16 \AA$ ), the micropore $(|x|>23 \AA$ ) and the (narrow) interface. The oxygen water density $\rho_{\mathrm{OW}}(x)=\iint \rho_{\mathrm{OW}}(x, y, z) \mathrm{d} y \mathrm{~d} z$ is reported in figure 6. It confirms that the particle and pore sizes are large enough to reach the "bulk" behaviour, both for the interlayer and the micropore, at least from the static point of view. In the interlayer, small oscillations in density are due to the location of the substitutions in the aluminosilicate sheet. This will become even more obvious in the case of cations; since most of the water molecules in the interlayer belong to the first or second coordination shells of the counterions, it is not surprising to also observe these density oscillations.

In the micropore, there is a strong layering at the particle surface, as indicated by the alternate oxygen and hydrogen distributions. Approximately two water layers are visible. This is due to the formation of $\mathrm{H}$-bonds between water and the $\mathrm{SiOH}, \mathrm{AlOH}$ and $\mathrm{AlOH}_{2}$ edge groups. Such a layering has already been observed by molecular simulation of water at the (001) surface of other minerals such as brucite, gibbsite, hydrotalcite, muscovite and talc [Wang et al., 2006]. Water structuring via H-bonding was also observed in water 
films confined between pyrophyllite edges [Churakov, 2007], but the range of the layering effect could not be evaluated due to the very thin water film studied, which however allowed to describe the proton exchanges between water and surface sites (not included in our classical description).

The ionic profiles are shown in figure 7 . The error bars represent $95 \%$ confidence interval as obtained by the block averaging method [Allen and Tildesley, 2002]. In the interlayer, the $\mathrm{Na}^{+}$and $\mathrm{Cs}^{+}$densities are modulated by the location of the substitutions in the mineral (also indicated in figure 7), whereas no anions are found. This latter point is an indication that thermal fluctuations do not allow anions to overcome the free energy barrier corresponding to their entrance into the interlayer $\left(\Delta \mathcal{F}_{2 \rightarrow 1}^{\#} \gg k_{B} T\right.$ for an anion in figure 2). In the micropore, both anion and cation densities reach a constant value near the center of the pore (edge of the simulation box), confirming that the box is sufficiently large, for this ionic strength, to reach the bulk behaviour. The $\mathrm{Na}^{+}$density displays a small maximum close to the surface $(x \sim \pm 18 \AA)$, which can be traced back to the susbtitutions near the edges (which are overall neutral), at approximately $5.3 \AA$ from the density maximum. The anion density also displays a maximum for $(x \sim \pm 20 \AA)$, most probably correlated to the cation density maximum. It will be of interest to see whether this still holds at lower ionic strength, or when the edges are deprotonated.

\subsection{Potential of Mean Force}

We already mentioned that the density can provide an estimate of the PMF, since Eq. (4) gives:

$$
\mathcal{F}(x)=-k_{B} T \ln \rho(x)+\mathcal{F}_{0}^{\prime \prime}
$$

The use of Eq. (5) is however limited to regions of the phase space that are correctly sampled with equilibrium simulations, i.e. that are accessible to the system via thermal fluctuations. This excludes regions of high free energy. Several routes exist to evaluate the PMF in such regions, most of them being related to (1) forcing the particle in the region of high PMF using a bias e.g. with umbrella sampling [Frenkel and Smit, 2002], (2) unbiasing the sampled quantity to reconstruct the PMF. We have used two such methods, namely the Weighted Histogram Analysis Method (WHAM) introduced by Kumar et al. [Kumar et al., 1995] and Umbrella Integration (UI), introduced by Kästner [Kästner and Thiel, 2005].

Both WHAM and UI are based on biased simulations where a tethering potential $V_{\text {bias }}^{i}(x)=0.5 k_{\text {bias }}\left(x-x_{0}^{i}\right)^{2}$ is applied on a test particle, whose position $x$ is sampled (umbrella sampling). This potential constrains the particle to remain around a position $x_{0}^{i}$. The tethering positions $\left\{x_{0}^{i}\right\}$ define a set of windows, and histograms for the (biased) probability $P_{b}^{i}(x)$ to be at a given value of $x$ are constructed for each window. WHAM and UI then use differently the same set $\left\{P_{b}^{i}(x)\right\}$ to reconstruct the unbiased PMF. In each window, the biased probability is given by

$$
P_{b}^{i}(x) \propto e^{-\left[\mathcal{F}(x)+V_{\text {bias }}^{i}(x)\right] / k_{B} T}
$$

and to recover the correct PMF it must be multiplied by a factor $\exp \left(+V_{\text {bias }}^{i}(x) / k_{B} T\right)$. Finally, the PMF $\mathcal{F}$ must be reconstructed to ensure consistency between all windows. 
This is achieved iteratively in the WHAM method by taking advantage of the overlap between histograms corresponding to neighbouring windows [Kumar et al., 1995, Roux, 1995]. In the UI method, the thermodynamic force $(-\nabla \mathcal{F})$ is estimated independently in each window, and the PMF is then obtained by numerical integration [Kästner and Thiel, 2005].

For umbrella sampling, we used 70 to 85 values (depending on the ion) inhomogeneously distributed between $x_{0}=3.0 \AA$ and $x_{0}=30.0 \AA$, more densely in the $14-25 \AA$ region to increase the accuracy where $\mathrm{PMF}$ variations are larger. The strength of the bias was $k_{\text {bias }}=20 \mathrm{kJmol}^{-1} \AA^{-2}$. This value is a compromise ensuring at the same time a strong restrain around the tethering positions $x_{0}^{i}$, and an overlap between the biased trajectories corresponding to $x_{0}^{i}$ and $x_{0}^{i+1}$. For each simulation, 50 ps of equilibration were followed by 300 ps of sampling. The computational cost of umbrella sampling is very high, since a relatively long simulation must be undertaken for each window around $x_{0}^{i}$, in order to correctly sample the biased probability $P_{b}^{i}(x)$. Nevertheless such an elaborate and expensive approach is unavoidable, as will be demonstrated below.

The uncertainty on the PMF as obtained by Eq. (5) can be estimated as $\sigma[\rho(x)] / \rho(x)$, where $\sigma$ is the width of the $95 \%$ confidence interval for $\rho(x)$ (see section 3.2). It is negligible in the case of water, but not for the ions. The error on the Umbrella Integration results can be evaluated using the method of Kästner and Thiel [Kästner and Thiel, 2006]. However the results are very small $\left(\sim 0.2 \mathrm{~kJ} / \mathrm{mol}\right.$, that is $\left.0.1 k_{B} T\right)$ compared to the variations of $\mathcal{F}$ (see below). We didn't estimate the uncertainty associated with the WHAM result. The comparison between the WHAM and UI results gives an indication on the overall accuracy of the biased schemes estimates.

\subsection{Results}

The PMF obtained with Eq. (5) is reported in figure 8 for water, $\mathrm{Na}^{+}, \mathrm{Cs}^{+}$and $\mathrm{Cl}^{-}$, for interlayer/micropore exchange on the right side of the clay particle $(x>0)$. For the ions, results of WHAM and UI are also shown. Since the PMF are determined up to an arbitrary constant -see Eq. (5)- we have chosen to set the PMF for each species to zero in the micropore. The large number of water molecules in the simulation box allows to obtain the density with sufficient statistical accuracy, therefore we did not undertake the more time-consuming umbrella sampling in that case. The absence of barrier for the interlayer/micropore exchange, and the very low activation energy $\Delta \mathcal{F}_{2 \rightarrow 1}^{\#}<k_{B} T$ indicates that thermal fluctuations allow to correctly sample the whole phase space.

The agreement between the estimate of Eq. (5) with both WHAM and UI is relatively good for $\mathrm{Na}^{+}$and $\mathrm{Cs}^{+}$, because the free energy difference is only a few $k_{B} T$ : Thermal fluctuations allow to overcome the barrier. This is why cation exchange events occur during the equilibrium simulation, so that the cation density is also relatively well sampled. The discrepancy observed for $\mathrm{Cs}^{+}$between the results of WHAM and UI on the one hand and Eq. (5) on the other hand can be traced back to the statistical inaccuracy on $\rho_{\mathrm{Cs}}(x)$, since the simulation box contains only $6 \mathrm{Cs}^{+}$ions. Furthermore, the result obtained by sampling the density only during the last 5 ns of the production run is closer to the WHAM and UI results (see figure 8c). This is an indication that the system might not be completely equilibrated with respect to $\mathrm{Cs}^{+}$density (before equilibration all $\mathrm{Cs}^{+}$ions 
were in the micropore). The requirement to use biased techniques to evaluate the PMF is even more obvious in the $\mathrm{Cl}^{-}$case: The result of Eq. (5) diverges around $x=13 \AA$, since no anion was found at a lower abscissa during the unbiased simulation.

For water and anions, the PMF is higher in the interlayer than in the micropore. The opposite is observed for cations. The free energy difference is about the thermal energy for water, and 2-3 $k_{B} T$ for cations, so that the barrier crossing occurs easily under thermal fluctuations. The free energy difference is however much larger for anions (8-10 $\left.k_{B} T\right)$, meaning that anions are excluded from the interlayer: The probability for an anion reaching the interface to enter into the interlayer is very small (of the order of $e^{-9} \sim$ $10^{-4}$ ). In addition, an anion that eventually crosses the interface has a high probability to cross it again and return into the micropore. Interestingly, up to the precision of our estimates (less than $1 k_{B} T$, see below), the interlayer to micropore transition is not activated for water and anions $\left(\Delta \mathcal{F}_{1 \rightarrow 2}^{\#} \sim 0\right.$ in figure 2). This result indicates that the transition probability for a diffusive particle reaching the interface from the interlayer is close to unity. The same holds for the transition from micropore to interlayer for cations $\left(\Delta \mathcal{F}_{2 \rightarrow 1}^{\#} \sim 0\right)$. We plan to investigate the effect of the deprotonation of the edge sites on these activation energies. Our results demonstrate the absence of activation barrier for the entrance of cations into the interlayer. This shows that the entropic penalty (excluded volume effect, as observed on the water potential of mean force) is more than compensated by the electrostatic energy gain.

An upper bound on the uncertainty on $\Delta \mathcal{F}_{1 \rightarrow 2}^{\#}$ can be estimated by the range of variations of $\mathcal{F}$ itself in each region (interlayer and micropore), which are larger than the uncertainties on each value of $\mathcal{F}(x)$, or the differences between the results of the various methods (Eq. (5), WHAM or UI). The final results are thus $\Delta \mathcal{F}_{1 \rightarrow 2}^{\#}(\mathrm{Na})=2.0 \pm 1.0 k_{B} T$ and $\Delta \mathcal{F}_{1 \rightarrow 2}^{\#}(\mathrm{Cs})=2.5 \pm 1.0 k_{B} T$. This upper limit is not negligible compared to the free energy differences. However this is partly due to the small value of the latter for these particular processes. For the anions, we have $\Delta \mathcal{F}_{2 \rightarrow 1}^{\#}(\mathrm{Cl})=9.0 \pm 1.0 k_{B} T$, so that the relative error is smaller.

\subsection{Discussion}

The study of the PMF allows to evaluate the free energy barriers $\Delta \mathcal{F}_{1 \rightarrow 2}^{\#}$ associated with the release of interlayer cations into the micropore of a porous clay sample. However only the free energy difference $\Delta \mathcal{F}_{1 \rightarrow 2}$ can be compared with experimental thermodynamic quantities. The free energy of ionic exchange (1) can be deduced from selectivity measurements on dispersed clays: Its value is $\Delta_{r} F^{\circ} \sim-4 \pm 2 k_{B} T$ (for a review and a discussion see Teppen and Miller [2006]). In this section we relate the PMF differences to $\Delta_{r} F^{\circ}$, before discussing the influence of the ionic strength on the activation barriers $\Delta \mathcal{F}_{1 \rightarrow 2}^{\#}$.

From the definition (5), the PMF difference is:

$$
\Delta \mathcal{F}_{1 \rightarrow 2}(i)=-k_{B} T \ln \frac{\rho_{\mathrm{ext}}(i)}{\rho_{\mathrm{int}}(i)}=-k_{B} T \ln \frac{C_{\mathrm{ext}}(i)}{C_{\mathrm{int}}(i)}
$$

While the first equality refers to numbers of tracers per unit of the reaction coordinate $\rho(x)$, that is a linear density, the second involves the species concentration $C_{\text {ext }}(i)$ in the 
micropore and $C_{\text {int }}(i)$ its volume concentration in the clay, and follows from $C_{\text {ext }} / C_{\text {int }}=$ $\rho_{\text {ext }} / \rho_{\text {int }}$.

Chosing the infinite dilution as reference state for the solution phase, and the bihydrated homoionic clay with counterions $i$ only $\left(i\right.$ being $\mathrm{Cs}^{+}$or $\mathrm{Na}^{+}$) for the clay phase, one can write the chemical potential in both phases as:

$$
\begin{aligned}
& \mu_{\text {ext }}(i)=\mu_{\text {ext }}^{\circ}(i)+k_{B} T \ln C_{\text {ext }}(i) \\
& \mu_{\text {int }}(i)=\mu_{\text {int }}^{\circ}(i)+k_{B} T \ln \alpha_{\text {int }}(i)
\end{aligned}
$$

with $\mu^{\circ}(i)$ the corresponding standard chemical potentials, and $\alpha_{\text {int }}(i)=C_{\text {int }}(i) / C_{\text {int }}^{\text {tot }}$ the ionic fraction of $i$ in the interlayer. The total charge density of counterions $C_{\mathrm{int}}^{\text {tot }}$ is fixed by the charge density of the mineral. The ideality assumption in Eq. (8a) is valid at low salt concentration in the micropore, while Eq. (8b) assumes ideality of the heteroionic clay (with both $\mathrm{Cs}^{+}$and $\mathrm{Na}^{+}$in the interlayer), i.e. that each ion in the interlayer behaves as in the corresponding bihydrated homoionic clay. This is a reasonable approximation for $\mathrm{Cs}^{+}$as was shown by molecular simulation [Marry and Turq, 2003]. Note that the reference state for $\mathrm{Cs}^{+}$is not experimentally stable.

At equilibrium, the free energy variation upon exchange of a tracer in the micropore with an interlayer $\mathrm{Na}^{+}$ion must vanish, so that:

$$
\mu_{\mathrm{int}}(\mathrm{Na})+\mu_{\mathrm{ext}}(\mathrm{Cs})=\mu_{\mathrm{int}}(\mathrm{Cs})+\mu_{\mathrm{ext}}(\mathrm{Na})
$$

From the definition (7) of $\Delta \mathcal{F}_{1 \rightarrow 2}(i)$ and Eqs. (8) we arrive at:

$$
\begin{aligned}
\Delta \mathcal{F}_{1 \rightarrow 2}(\mathrm{Na})-\Delta \mathcal{F}_{1 \rightarrow 2}(\mathrm{Cs}) & =\left[\mu_{\text {ext }}^{\circ}(\mathrm{Cs})+\mu_{\text {int }}^{\circ}(\mathrm{Na})\right]-\left[\mu_{\text {int }}^{\circ}(\mathrm{Cs})+\mu_{\text {ext }}^{\circ}(\mathrm{Na})\right] \\
& =\Delta_{r} F^{\circ}
\end{aligned}
$$

According to the simulation results, the overall ionic exchange between a bihydrated Naclay and a bihydated Cs-Clay has an overall exchange free energy $\Delta_{r} F^{\circ} \sim 0(-0.5 \pm$ $\left.2.0 k_{B} T\right)$. A possible source of discrepancy with the experimental value could lie in the ideality assumption made in the present analysis, but the main difference might be found in the clay water content. The exchange of ions in the bihydrated state is representative of a porous clay material, which is the relevant one for example in the context of waste confinement by clay barriers, but not necessarily of a clay dispersed in water, which corresponds to the experimental determination of selectivity. A detailed comparison of the exchange free energy with the experimental results would involve the difference of swelling free energies of bihydrated $\mathrm{Na}$ and Cs-clays (the latter being not stable). However this is not the purpose of the present paper, which focusses on the interface crossing kinetics.

We now turn to the influence of the salt concentration in the micropore on the kinetics of interlayer cations release. When considering the exchange of a $\mathrm{Cs}^{+}$tracer with a Naclay, one can approximate $C_{\text {int }}(\mathrm{Na})$ by $C_{\text {int }}^{\text {tot }}$, and $C_{\text {ext }}(\mathrm{Na})$ by the ionic strength $C_{\text {ext }}^{\text {tot }}$ in the micropore. Then Eq. (7) results in:

$$
\Delta \mathcal{F}_{1 \rightarrow 2}(\mathrm{Na}) \approx-k_{B} T \ln \frac{C_{\mathrm{ext}}^{\mathrm{tot}}}{C_{\mathrm{int}}^{\mathrm{tot}}}
$$

The PMF profiles have demonstrated that $\Delta \mathcal{F}_{2 \rightarrow 1}^{\#} \sim 0$ for the transfer of cations from the micropore into the interlayer, so that the activation barrier $\Delta \mathcal{F}_{1 \rightarrow 2}^{\#}$ is equal to $\Delta \mathcal{F}_{1 \rightarrow 2}$. 
For a neutral edge, the interactions of the ions with the interface should not depend on the external ionic concentration (this won't be true for a charged edge), so that the absence of barrier $\Delta \mathcal{F}_{2 \rightarrow 1}^{\#}$ is most probably preserved at other ionic strength. Therefore we can expect the transition from the interlayer to the micropore to be less activated as the ionic strength increases, and from Eqs. (10) and (11) the barrier should vary as:

$$
\Delta \mathcal{F}_{1 \rightarrow 2}^{\#}(\mathrm{Cs}) \approx-\Delta_{r} F^{\circ}-k_{B} T \ln \frac{C_{\mathrm{ext}}^{\mathrm{tot}}}{C_{\mathrm{int}}^{\mathrm{tot}}}
$$

The probability for an interlayer cation reaching the particle edge to be released into the micropore should thus increase with increasing ionic strength. Under real conditions the external cation concentration $C_{\text {ext }}^{\text {tot }}$ is always lower than $C_{\text {int }}^{\text {tot }}$ so that (12) indeed corresponds to an activation barrier.

We have shown that microscopic simulations allow to evaluate the PMF for the interlayer/micropore exchange which controls the transition probabilities for water molecules and ions reaching the particle/pore interface. All the results are in agreement with the common sense on ionic exchange and anion exclusion. Additional studies are however necessary to provide informations on the origin of the PMF, and the relative contribution of e.g. electrostatic and steric effects. We now turn to the other aspect of the exchange dynamics, namely the evaluation of diffusion coefficients.

\section{Diffusion coefficients}

\subsection{Method}

To complete the justification of the simple model depicted in figure 1 (2D-diffusion $D_{\text {in }}$ in the interlayer, 3D-diffusion $D_{\text {ext }}$ in the micropore), one needs to evaluate the components of the diffusion tensor $\mathbb{D}=\operatorname{Diag}\left(D_{x x}, D_{y y}, D_{z z}\right)$. Each component is in fact a function of the position $x$, so that a special trajectory analysis is necessary. We have used the method thoroughly described in [Liu et al., 2004], for which fictitious layer boundaries are introduced. We recall here only the main aspects of this method. The simulation box is divided in windows (around a position $x_{i}$ ), and $D_{y y}$ (resp. $D_{z z}$ ) is determined from the mean-square displacement $\left\langle\Delta y_{i}^{2}(t)\right\rangle$ along $y$ (resp. $z$ ) of particles remaining in a given window and the survival probability in the window $\mathcal{P}_{i}(t)$ :

$$
D_{y y}\left(x_{i}\right)=\lim _{t \rightarrow \infty} \frac{\left\langle\Delta y_{i}^{2}(t)\right\rangle}{2 t \mathcal{P}_{i}(t)}
$$

The $D_{x x}$ component can be obtained from the autocorrelation of the functions:

$$
\Psi_{n}^{i}(t)=\sin \left(n \pi \frac{x(t)-x_{\min }^{i}}{x_{\max }^{i}-x_{\min }^{i}}\right)
$$

where $x_{\text {min }}^{i}$ and $x_{\text {max }}^{i}$ define the sampling window $i$ of width $L=x_{\text {max }}^{i}-x_{\text {min }}^{i}$ (we used $L=4 \AA$ for all windows), and $n$ is an integer. When the PMF is constant, one can 
show that the autocorrelation $\left\langle\Psi_{n}^{i}(t) \Psi_{n}^{i}(0)\right\rangle$ decays as $\exp \left(-(n \pi / L)^{2} D_{x x} t\right)$, so that $D_{x x}$ is obtained by:

$$
D_{x x}\left(x_{i}\right)=-\left(\frac{L}{n \pi}\right)^{2} \lim _{t \rightarrow 0} \frac{\ln \left\langle\Psi_{n}^{i}(t) \Psi_{n}^{i}(0)\right\rangle}{t}
$$

We restricted our study of the diffusion coefficient to regions where the PMF is constant (interlayer and micropore, not the interface) because when it isn't the diffusion coefficient must be determined from the autocorrelation of a function involving the PMF, and the latter must therefore be known with great accuracy. Furthermore, the PMF varies in our case very sharply, over a distance of the order of the window width necessary to perform the sampling, and this reinforces the first difficulty. Comparison of the results obtained for $D_{x x}$ with different eigenfunctions $\Psi_{n}(n=1,2,3)$ confirms that the motion is indeed diffusive in the studied regions. Since the larger $n$, the faster $\left\langle\Psi_{n}(t) \Psi_{n}(0)\right\rangle$ decays (and the faster the values become noisy), the best estimates for $D_{x x}$ are obtained with $n=1$, which are the ones reported below.

\subsection{Results}

The components of the water diffusion tensor are shown in figure 9 as a function of position $x$ in the interlayer and in the micropore, where the potential of mean force $\mathcal{F}(x)$ is constant, but not in the transition region. In the interlayer, the diffusion is twodimensional $\left(D_{z z} \sim 0\right.$, not shown) and almost isotropic in the $x y$-plane $\left(D_{x x} \sim D_{y y}\right)$. Furthermore, the value of $D_{x x}$ and $D_{y y}$ only slightly depend on position, and is close to $0.9 \pm 0.110^{-9} \mathrm{~m}^{2} \mathrm{~s}^{-1}$. These observations are in agreement with MD simulations of "bulk" interlayers [Marry and Turq, 2003, Malikova et al., 2004a] and experimental determination by Quasielastic Neutron Scattering (QENS) [Malikova et al., 2005, 2006]. This confirms that although short with respect to real clay particles, the one used in our simulation is large enough to account for the "bulk" clay behaviour.

In the micropore, diffusion along the clay particle $\left(D_{y y} \sim D_{z z}\right)$ is similar to bulk water diffusion: The value $2.5 \pm 0.210^{-9} \mathrm{~m}^{2} \mathrm{~s}^{-1}$ is in agreement both with experimental results $\left(2.3 \pm 0.110^{-9} \mathrm{~m}^{2} \mathrm{~s}^{-1}\right)$ [Krynicki et al., 1978] and MD simulations of bulk SPC/E water $\left(D^{\mathrm{SPC} / \mathrm{E}}=2.4 \pm 0.110^{-9} \mathrm{~m}^{2} \mathrm{~s}^{-1}\right)$ [van der Spoel et al., 1998, Guillot, 2002]. Interestingly, the component normal to the particle surface is smaller than the two others: $D_{x x} \sim 1.5 \pm$ $0.110^{-9} \mathrm{~m}^{2} \mathrm{~s}^{-1}$. The influence of the surface on diffusion thus appears to be longer-ranged than that on density (which has already reached its bulk value). A larger simulation box should allow to observe the transition from the observed $D_{x x}$ values close to the surface (a few $\mathrm{nm}$ ) to the bulk value $D^{\mathrm{SPC} / \mathrm{E}}$ at larger distances. Work is in progress to address this point.

For $\mathrm{Na}^{+}, D_{x x} \sim D_{y y}$ is almost constant in the interlayer, with $D_{i n}^{N a} \sim 0.6 \pm 0.210^{-9} \mathrm{~m}^{2} \mathrm{~s}^{-1}$ in agreement with previous simulations of $\mathrm{Na}$-montmorillonite in the dihydrated state [Marry and Turq, 2003]. In the micropore, $D_{x x} \sim D_{y y} \sim D_{z z}$ with $D_{e x t}^{N a} \sim 1.2 \pm 0.210^{-9} \mathrm{~m}^{2} \mathrm{~s}^{-1}$, in agreement with the experimental determination [Mills and Lobo, 1989] and with simulation results [Koneshan et al., 1998] in a bulk electrolyte solution. There is apparently no anisotropy for $\mathrm{Na}^{+}$diffusion in the micropore, in contrast with water. For $\mathrm{Cs}^{+}$and $\mathrm{Cl}^{-}$, the number of ions in the simulation box is too small to provide a fair estimate of the diffusion coefficients. 


\section{Conclusion}

We have used Molecular Dynamics simulations to investigate the exchange of water and ions between interlayer and micropores in clays. A force field was first developed to describe the clay particle edges, based on DFT calculations. Classical MD simulations were then performed to evaluate the free energy profile and the diffusion coefficients along a selected reaction coordinate. The results confirm the generally admitted ideas of ionic exchange and anion exclusion, and allow for a more quantitative description of these processes. Improvements in the force field determination are desirable: Use of bigger cells, relaxation of the edge atoms (in the presence of water), allowance for $\mathrm{OH}$ bonds rotation. The same strategy (charge-fitting from ab-initio simulation) could also be adopted with edges other than the (010) studied here, which is not the only stable one, or with deprotonated edge sites, to indirectly study the influence of $\mathrm{pH}$ on the exchange.

From a practical point of view, the main conclusion of the present work is that simple models for water and cation transport such as the one illustrated in figure 1 are relevant, provided that the appropriate diffusion tensor and transition probabilities at the particle/pore interface are used. The latter can be obtained using molecular simulations and the approach developed in this paper. The fact that the exchange of water between interlayer and micropore is not activated justifies the assumption underlying the averaging procedure introduced by Bourg et al. [Bourg et al., 2006], which has proved to be very efficient in interpreting macroscopic tracer diffusion data in compacted, water-saturated bentonite.

A final caveat must be expressed on the choice of the sole position as the reaction coordinate to describe the exchange. We already mentioned in section 3.1 that a subtle quantity such as the coordination number of the transiting molecule or ion cannot be ruled out. Therefore one must keep in mind that the quantitative results given in this paper provide a lower bound on the free energy barriers. Further studies are necessary to describe the microscopic mechanisms accompanying the transition in more details, and we are planning to investigate the effect of the change in coordination number upon entering into or leaving the interlayer. Experimental techniques such as neutron scattering allow to probe the clay-water interface on the atomistic scale. However the investigation of the lateral surfaces of clay particles (edges) is difficult since the latter represent only a small fraction of the total clay-water interface, which includes the interlayer spaces and the basal surfaces of clay particles. Therefore molecular simulation is a valuable tool to study the processes occuring on the lateral surfaces [Churakov, 2007].

It will be of particular interest to investigate the influence of several factors on the interlayer/micropore transition: Ionic strength in the micropore, protonation state of the edge (which is controlled by the $\mathrm{pH}$ ), interlayer water content, or presence of multivalent

cations $\left(\mathrm{Ca}^{2+}\right)$. It has indeed been argued that such cations might favour the entrance of ionic pairs $\left(\mathrm{CaCl}^{+}\right)$[Ferrage et al., 2005b]. Work in these directions is under progress. 


\section{Acknowledgements}

We would like to thank Jean-François Dufrêche and Olivier Benichou for useful discussions, as well as Zhenhao Duan and two anonymous reviewers for their suggestions to improve the present article. BR acknowlegdes financial support from ANDRA (Agence Nationale pour la Gestion des Déchets Radioactifs). Financial support of GdR PARIS (Physico-chimie des Actinides et autres Radioéléments aux Interfaces et en Solution) is gratefully acknowledged. LI2C is part of the SFR Sciences Chimiques pour l'Analyse et la Mesure de Paris-Centre.

\section{References}

CPMD. Copyright IBM Corp 1990-2001, Copyright MPI für Festkörperforschung Stuttgart, 1997-2004.

M.P. Allen and D.J. Tildesley. Computer simulation of Liquids. Oxford Science Publications, 2002.

G.B. Bachelet, D.R.Hamann, and M. Schlüter. Pseudopotentials that work: From H to Pu. Phys. Rev. B, 26(8):4199-4228, 1982.

A.D. Becke. Density-functional exchange-energy approximation with correct asymptotic behavior. Phys. Rev. A, 38(6):3098-3100, 1988.

H.J.C Berendsen, J.R. Grigera, and T.P. Straatsma. The missing term in effective pair potentials. J. Phys. Chem., 91(24):6269-6271, 1987.

B.R. Bickmore, K.M. Rosso, K.L. Nagy, R.T. Cygan, and C.J. Tadanier. Ab initio determination of edge surface structures for dioctahedral $2: 1$ phyllosilicates: Implications for acid-base reactivity. Clay Clay Miner., 51(4):359-371, 2003.

E.S. Boek, P.V. Coveney, and N.T. Skipper. Monte carlo molecular modelling studies of hydrated Li-, Na- and K-smectites: Understanding the role of potassium as a clay swelling inhibitor. J. Am. Chem. Soc., 117(50):12608-12617, 1995a.

E.S. Boek, P.V. Coveney, and N.T. Skipper. Molecular modeling of clay hydration: a study of hysteresis loops in the swelling curves of sodium montmorillonites. Langmuir, 11(12):4629-4631, 1995b.

I.C. Bourg, G. Sposito, and A.C.M. Bourg. Tracer diffusion in compacted, water-saturated bentonite. Clay Clay Miner., 54(3):363-374, 2006.

M. Bradbury and B. Baeyens. Near field sorption data bases for compacted MX-80 bentonite for performance assessment of a high-level radioactive waste repository in Opalinus clay host rock. Technical report, Paul Scherrer Institut, 2003.

R. Calvet. Hydratation de la montmorillonite et diffusion des cations compensateurs. Ann. Agron., 24:77-217, 1973. 
F.-R.C. Chang, N.T. Skipper, and G. Sposito. Computer simulation of interlayer molecular structure in sodium montmorillonite hydrates. Langmuir, 11(7):2734-2741, 1995.

F.-R.C. Chang, N.T. Skipper, and G. Sposito. Monte-Carlo and molecular dynamics simulations of interfacial structure in Li-montmorillonite hydrates. Langmuir, 13(7): 2074-2082, 1997.

F.-R.C. Chang, N.T. Skipper, and G. Sposito. Monte-Carlo and molecular dynamics simulations of electrical double-layer structure in potassium- montmorillonite hydrates. Langmuir, 14(5):1201-1207, 1998.

S.V. Churakov. Ab initio study of sorption on pyrophyllite: Structure and acidity of the edge sites. J. Phys. Chem. B., 110(9):4135-4146, 2006.

S.V. Churakov. Structure and dynamics of the water films confined between edges of pyrophyllite: A first principle study. Geochim. Cosmochim. Acta, 71(5):1130-1144, 2007.

R.T. Cygan, J.-J. Liang, and A.G. Kalinichev. Molecular models of hydroxide, oxohydroxide and clay phases and the development of a general force field. J. Phys. Chem. B, 108(4):1255-1266, 2004.

A.V.C. de Siqueira, N.T. Skipper, P.V. Coveney, and E.S. Boek. Computer simulation evidence for enthalpy driven dehydration of smectite clays at elevated pressures and temperatures. Mol. Phys., 92(1):1-6, 1997.

A. Delville. Structure and properties of confined liquids: A molecular model of the claywater interface. J. Phys. Chem., 97(38):9703-9712, 1993.

A. Delville. Structure of liquids at a solid interface: An application to the swelling of clay by water. Langmuir, 8(7):1796-1805, 1992.

E. Ferrage, B. Lanson, N. Malikova, A.Plancon, B.A. Sakharov, and V.A. Drits. New insights on the distribution of interlayer water in bi-hydrated smectite from X-ray diffraction profile modeling pf 001 reflections. Chem. Mater., 17(13):3499-3512, 2005a.

E. Ferrage, C. Tournassat, E. Rinnert, L. Charlet, and B. Lanson. Experimental evidence for Ca-chloride ion pairs in the interlayer of montmorillonite. an XRD profile modeling approach. Clay Clay Miner., 53(4):348-360, 2005b.

D. Frenkel and B. Smit. Understanding Molecular Simulations, From Algorithms to Applications. Academic Press, 2002.

M.H. Fu, Z.Z. Zhang, and P.F. Low. Changes in the properties of a montmorillonite-water system during the adsorption and desorption of water: Hysteresis. Clay Clay Miner., 38(5):485-492, 1990.

J.A. Greathouse and R.T. Cygan. Molecular dynamics simulation of uranyl(vi) adsorption equilibria onto an external montmorillonite surface. Phys. Chem. Chem. Phys., 7(20): 3580-3586, 2005. 
J.A. Greathouse and R.T. Cygan. Water structure and aqueous uranyl(vi) adsorption equilibria onto external surfaces of beidellite, montmorillonite, and pyrophyllite: Results from molecular simulations. Env. Sci. Technol., 40(12):3865-3871, 2006.

B. Guillot. A reappraisal of what we have learnt during three decades of computer simulations of water. J. Mol. Liq., 101:219-260, 2002.

E.J.M. Hensen and B. Smit. Why clays swell. J. Phys. Chem. B, 106(49):12664-12667, 2002.

E.J.M. Hensen, T.J. Tambach, A. Bliek, and B. Smit. Adsorption isotherms of water in Li-, Na- and K-montmorillonite by molecular simulation. J. Chem. Phys., 115(7): 3322-3329, 2001.

T. Hiemstra, P. Venema, and W.H. VanRiemsdijk. Intrinsic proton affinity of reactive surface groups of metal (hydr)oxides: The bond valence principle. J. Coll. Interf. Sci., 184(2):680-692, 1996.

J. Kästner and W. Thiel. Bridging the gap between thermodynamic integration and umbrella sampling provides a novel analysis method: "umbrella integration". J. Chem. Phys., 123(14):144104, 2005.

J. Kästner and W. Thiel. Analysis of the statistical error in umbrella sampling simulations by umbrella integration. J. Chem. Phys., 124:234106, 2006.

H. Kato, M. Muroi, N. Yamada, H. Ishida, and H. Sato. Scientific basis for nuclear waste management, volume 18. Materials Research Society, Pennsylvania, 1995.

B. Kirchner and J. Hutter. Solvent effects on electronic properties from wannier functions in a dimethyl sulfoxide/water mixture. J. Chem. Phys., 121(11):5133-5142, 2004.

R.J. Kirkpatrick, A.G. Kalinichev, and J.W. Wang. Molecular dynamics modelling of hydrated mineral interlayers and surfaces: structure and dynamics. Miner. Mag., 69 (3):289-308, 2005.

L. Kleinman and D.M. Bylander. Efficacious form for model pseudopotentials. Phys. Rev. Lett., 48(20):1425-1428, 1982.

S. Koneshan, C. Rasaiah, R.M. Lynden-Bell, and S.H. Lee. Solvent structure, dynamics, and ion mobility in aqueous solutions at $25^{\circ}$ C. J. Phys. Chem. B, 102(21):4193-4204, 1998.

K. Krynicki, C.D. Green, and D.W. Sawyer. Pressure and temperature dependence of self-diffusion in water. Faraday Discuss. Chem. Soc., 66:199-208, 1978.

S. Kumar, J.M. Rosenberg, D. Bouzida, R.H. Swendsen, and P.A. Kollman. Multidimensional free-energy calculations using the weighted histogram analysis method. J. Comp. Chem., 16(11):1339-1350, 1995. 
A. Laio and M. Parrinello. Escaping free-energy minima. Proc. Nat. Acad. Sci., 99(20): 12562-12566, 2002.

A. Laio, J. VandeVondele, and U. Rothlisberger. D-RESP: Dynamically generated electrostatic potential derived charges from quantum mechanics/molecular mechanics simulations. J. Phys. Chem. B, 106(29):7300-7307, 2002.

C. Lee, W. Yang, and R.G. Parr. Development of the Colle-Salvetti correlation-energy formula into a functional of the electron density. Phys. Rev. B, 37(2):785-789, 1988.

P. Liu, E. Harder, and B.J. Berne. On the calculation of diffusion coefficients in confined fluids and interfaces with an application to the liquid-vapor interface of water. J. Phys. Chem. B, 108(21):6595-6602, 2004.

X.-D. Liu and X.-C. Lu. A thermodynamic understanding of clay-swelling inhibition by potassium ions. Angew. Chem. Int. Ed., 45(38):6300-6303, 2006.

N. Malikova, V. Marry, J.-F. Dufrêche, C. Simon, P. Turq, and E. Giffaut. Temperature effect in a montmorillonite clay at low hydration - microscopic simulation. Mol. Phys., 102(18):1965-1977, 2004a.

N. Malikova, V. Marry, J.-F. Dufrêche, and P. Turq. Na/Cs - montmorillonite: temperature activation of diffusion by simulation. Curr. Opinion in Coll. Int. Sci., 9(1-2): 124-127, 2004b.

N. Malikova, A. Cadène, V. Marry, E. Dubois, P. Turq, J.-M. Zanotti, and S. Longeville. Diffusion of water in clays - microscopic simulation and neutron scattering. Chem. Phys., 317(2-3):226-235, 2005.

N. Malikova, A. Cadène, V. Marry, E. Dubois, and P. Turq. Diffusion of water in clays on the microscopic scale: modeling and experiments. J. Phys. Chem. B, 110(7):3206-3214, 2006.

L. Maragliano, A. Fischer, E. Vanden-Eijnden, and G. Ciccotti. String method in collective variables: Minimum free energy paths and isocommittor surfaces. J. Chem. Phys., 125 (2):024106, 2006.

V. Marry and P. Turq. Microscopic simulations of interlayer structure and dynamics in bihydrated heteroionic montmorillonites. J. Phys. Chem. B, 107(8):1832-1839, 2003.

V. Marry, P. Turq, T. Cartailler, and D. Levesque. Microscopic simulation for structure and dynamics of water and counterions in a monohydrated montmorillonite. J. Chem. Phys., 117(7):3454-3463, 2002.

G.J. Martyna and M.E. Tuckerman. A reciprocal space based method for treating long range interactions in ab initio and force-field-based calculations in clusters. J. Chem. Phys., 110(6):2810-2821, 1999.

R. Mills and V.M.M. Lobo. Self-diffusion in Electrolyte Solutions. Physical Sciences Data 36. Elsevier, 1989. 
S.H. Park and G. Sposito. Monte-Carlo simulation of total radial distribution functions for interlayer water in Li-, Na- and K-montmorillonite. J. Phys. Chem. B, 104(19): 4642-4648, 2000.

B. Roux. The calculation of the potential of mean force using computer simulations. Comput. Phys. Comm., 91(1-3):275-282, 1995.

R.M. Shroll and D.E. Smith. Molecular dynamics simulations in the grand canonial ensemble: Application to clay mineral swelling. J. Chem. Phys., 111(19):9025-9033, 1999.

N.T. Skipper, K. Refson, and J.D.C. McConnell. Computer calculation of water-clay interactions using atomic pair potentials. Clay Minerals, 24(2):411-425, 1989.

N.T. Skipper, F.-R.C. Chang, and G. Sposito. Monte-Carlo simulation of interlayer molecular-structure in swelling clay-minerals, 1. Methodology. Clay Clay Miner., 43 (3):285-293, 1995a.

N.T. Skipper, G. Sposito, and F.-R.C. Chang. Monte carlo simulation of interlayer molecular structure in swelling clay minerals, 2. Monolayer hydrates. Clay Clay Miner., 43 (3):294-303, 1995b.

D.E. Smith. Molecular computer simulations of the swelling properties and interlayer structure of cesium montmorillonite. Langmuir, 14(20):5959-5967, 1998.

D.E. Smith, Y. Wang, A. Chaturvedi, and H.D. Whitley. Molecular simulations, of the pressure, temperature, and chemical potential dependencies of clay swelling. J. Phys. Chem. B, 110(40):20046-20054, 2006.

G. Sposito, N.T. Skipper, R. Sutton, S.H. Park, A.K. Soper, and J.A. Greathouse. Surface geochemistry of the clay minerals. Proc. Nat. Acad. Sci., 96(7):3358-3364, 1999.

R. Sutton and G. Sposito. Molecular simulation of interlayer structure and dynamics in $12.4 \AA$ Cs-smectite hydrates. J. Coll. Interf. Sci., 237(2):174-184, 2001.

Dossier 2005 Argile : Synthèse. Évaluation de la faisabilité du stockage géologique en formation argileuse. Technical report, ANDRA, Châtenay-Malabry, 2005.

T.J. Tambach, P.G. Bolhuis, and B. Smit. A molecular mechanism of hysteresis in clay swelling. Angew. Chem. Int. Ed., 43(20):2650-2652, 2004 a.

T.J. Tambach, E.J.M. Hensen, and B. Smit. Molecular simulations of swelling clay minerals. J. Phys. Chem. B, 108(23):7586-7596, 2004 b.

T.J. Tambach, P.G. Bolhuis, E.J.M. Hensen, and B. Smit. Hysteresis in clay swelling induced by hydrogen bonding: Accurate prediction of swelling states. Langmuir, 22(3): 1223-1234, 2006.

B.J. Teppen and D.M. Miller. Hydration energy determines isovalent cation exchange selectivity by clay minerals. Soil Sci. Soc. Am. J., 70(1):31-40, 2006. 
J.O. Titiloye and N.T. Skipper. Molecular dynamics simulation of methane in sodium montmorillonite clay hydrates at elevated pressures and temperatures. Mol. Phys., 99 (10):899-906, 2001.

C. Tournassat, E. Ferrage, C. Poinsignon, and L. Charlet. The titration of clay minerals II. Structure-based model and implications for clay reactivity. J. Coll. Interf. Sci., 273 (1):234-246, 2004.

N. Troullier and J.L. Martins. Efficient pseudopotentials for plane-wave calculations. Phys. Rev. B, 43(3):1993-2006, 1991.

D. van der Spoel, P.J. van Maaren, and H.J.C. Berendsen. A systematic study of water models for molecular simulation: Derivation of water models optimised for use with a reaction field. J. Chem. Phys., 108(24):10220-10230, 1998.

J.W. Wang, A.G. Kalinichev, and R.J. Kirkpatrick. Molecular modeling of water structure in nano-pores between brucite (001) surfaces. Geochim. Cosmochim. Acta, 68(16):33513365, 2004.

J.W. Wang, A.G. Kalinichev, R.J. Kirkpatrick, and R.T. Cygan. Structure, energetics and dynamics of water adsorbed on the muscovite (001) surface: A molecular dynamics simulation. J. Phys. Chem. B, 109(33):15893-15905, 2005.

J.W. Wang, A.G. Kalinichev, and R.J. Kirkpatrick. Effects of substrate structure and composition on the structure, dynamics and energetics of water on mineral surfaces: a molecular dynamics modeling study. Geochim. Cosmochim. Acta, 70(3):562-582, 2006.

H.D. Whitley and D.E. Smith. Free energy, energy, and entropy of swelling in Cs-, Na-, and Sr-montmorillonite clays. J. Chem. Phys., 120(11):5387-5395, 2004.

D.A. Young and D.E. Smith. Simulations of clay mineral swelling and hydration: Dependence upon interlayer ion size and charge. J. Phys. Chem. B, 104(39):9163-9170, 2000. 
Figure 1: Conceptual model for the dynamics of exchange between interlayer and micropore. Diffusion is in 2D in the interlayer, and 3D in the pore. A diffusing particle reaching the interface will cross it with a probability controlled by the free energy profile along the interface (see text). The arrows represent the interface crossing reactions.

Figure 2: Free energy profile along a reaction coordinate: In order to go from a state 1 (e.g. characterizing a given molecule in the interlayer), to a state 2 (e.g. characterizing the same molecule in the micropore), the system must in principle overcome a barrier $\Delta \mathcal{F}_{1 \rightarrow 2}^{\#}$. The reverse reaction occurs if the system overcomes the barrier $\Delta \mathcal{F}_{2 \rightarrow 1}^{\#}$. The overall free energy change is $\Delta \mathcal{F}_{1 \rightarrow 2}$.

Figure 3: Snapshot of the simulation box. $\mathrm{Na}^{+}$ions are in blue, $\mathrm{Cs}^{+}$in orange, and $\mathrm{Cl}^{-}$in pink. Clay $\mathrm{Al}$ atoms are in green, $\mathrm{Si}$ in yellow, $\mathrm{O}$ in red and $\mathrm{H}$ in white. Water molecules are in gray.

Figure 4: Detail of the edge [010] structure. Si tetrahedra end with an $\mathrm{SiOH}$ bond (top and bottom), whereas $\mathrm{Al}$ octahedra end either with $\mathrm{AlOH}$ (top) or $\mathrm{AlOH}_{2}$ (bottom) sites.

Figure 5: Two-dimensional density $\rho_{\mathrm{OW}}(x, z)$ of water oxygens. The darker regions correspond to lower density. The arrows represent the trajectories of water molecules, initially in the micropore, pulled along the $x$-direction into the particle: They find their way into the interlayer whatever their initial $z$-position.

Figure 6: Density profiles of water oxygens (solid line) and hydrogens (dotted line) across the simulation box. The hydrogen density was divided by a factor of two. The densities are almost constant in the interlayer and in the micropore. Close to the particle surface, there is a strong layering of the solvent (approximately for $16 \AA \leq|x| \leq 23 \AA$ ).

Figure 7: Density profiles of $\mathrm{Na}^{+}$(solid line), $\mathrm{Cs}^{+}$(dashed line) and $\mathrm{Cl}^{-}$(dotted line) across the simulation box. The location of the $\mathrm{Al}$ to $\mathrm{Mg}$ substitutions in the mineral layer is also reported (o). 
Figure 8: Potential of mean force corresponding to the interlayer/micropore exchange on the right side of the clay particle $(x>0)$, divided by the thermal energy. For water (a), only the estimate of Eq. (5) is reported (dotted line). For $\mathrm{Na}^{+}$(b), $\mathrm{Cs}^{+}$(c) and $\mathrm{Cl}^{-}$(d), the PMF as obtained by and Umbrella Integration (solid line) and Weighted Histogram Analysis Method (dashed line) are also shown. Note the different scales used.

Figure 9: Components of the water diffusion tensor as a function of position, in the interlayer and in the micropore. In the interlayer, $D_{x x} \sim D_{y y}$ (and $D_{z z} \sim 0$ ), whereas in the micropore $D_{x x} \neq D_{y y} \sim D_{z z}$. The diffusion coefficient along the particle surface $\left(D_{y y} \sim D_{z z}\right)$ is larger than perpendicular to the surface. The water potential of mean force is also reported as a dotted line. 
Electronic Annex 1: Atomic coordinates (including clay atoms, ions and water molecules). This file is a DLPOLY CONFIG file (http://www.cse.scitech.ac.uk/ccg/software/DL_POLY/), with a header containing the simulation box size.

Electronic Annex 2: Atomic partial charges and Lennard-Jones parameters. This file is a DLPOLY FIELD file (http://www.cse.scitech.ac.uk/ccg/software/DL_POLY/). The order of atoms corresponds to that in electronic annex EA-1. 
Figure 1

\begin{tabular}{|c|c|c|}
\hline $\begin{array}{c}\text { Pore } \\
(3 D)\end{array}$ & $\frac{\text { Particle }}{(2 D)}$ & $\begin{array}{c}\text { Pore } \\
(3 D)\end{array}$ \\
$D_{\text {ext }}$ & $D_{\text {in }}$ & $D_{\text {ext }}$ \\
& $\rightarrow$ \\
\hline
\end{tabular}


Figure 2

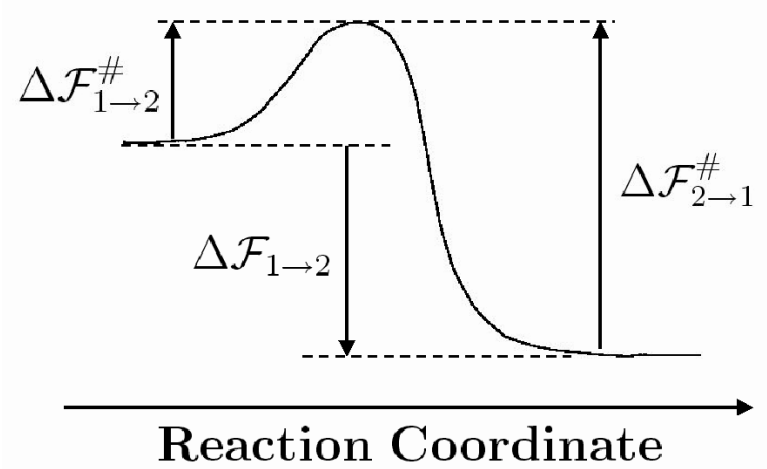


Figure 3

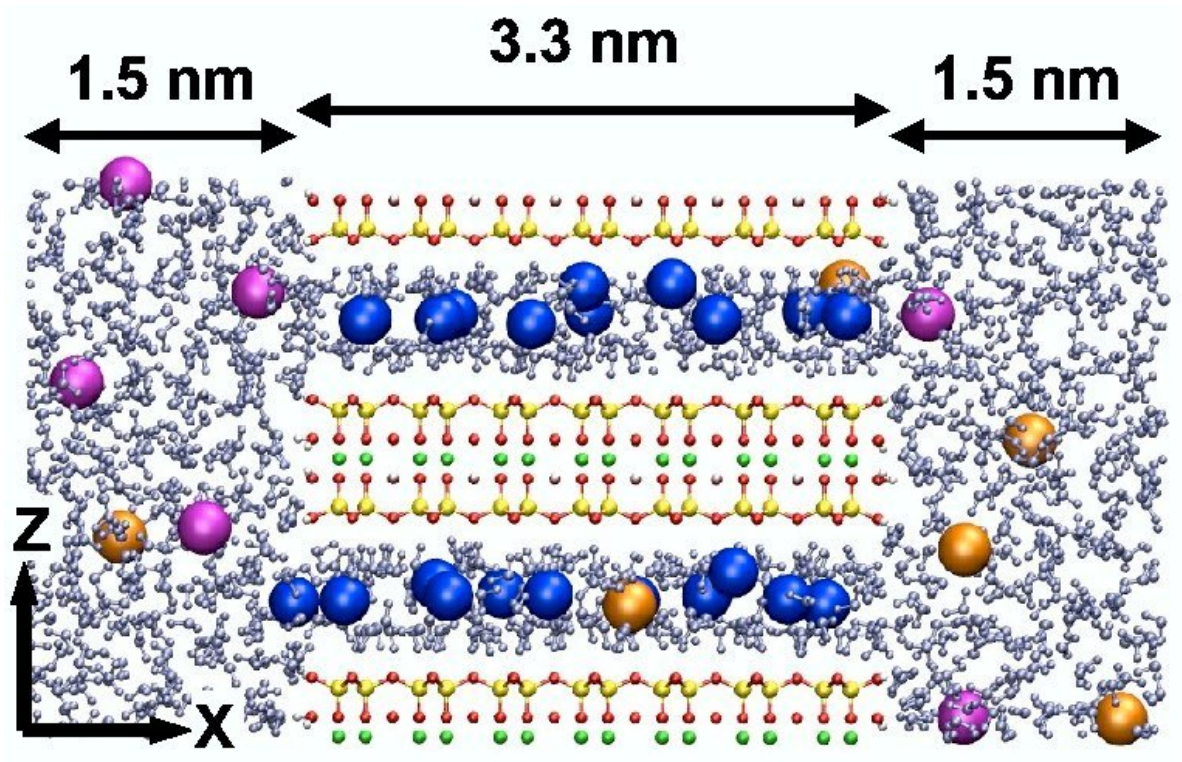


Figure 4

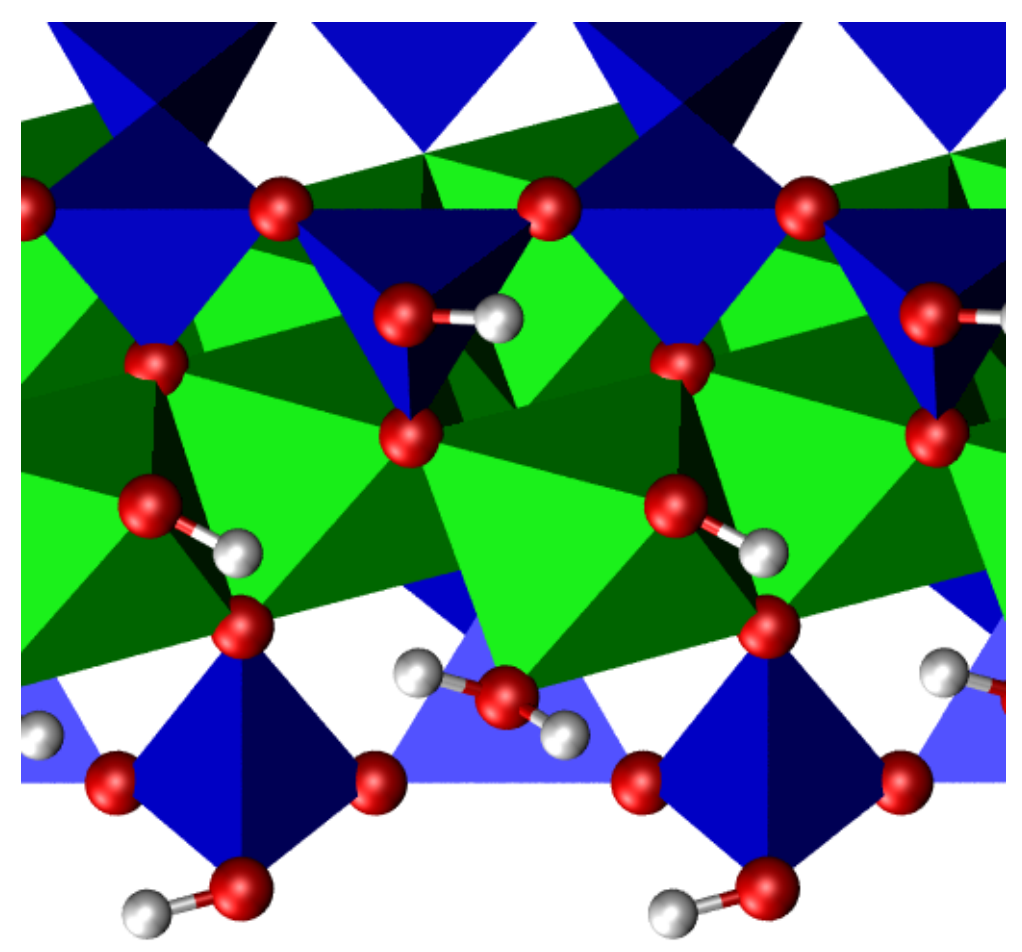


Figure 5

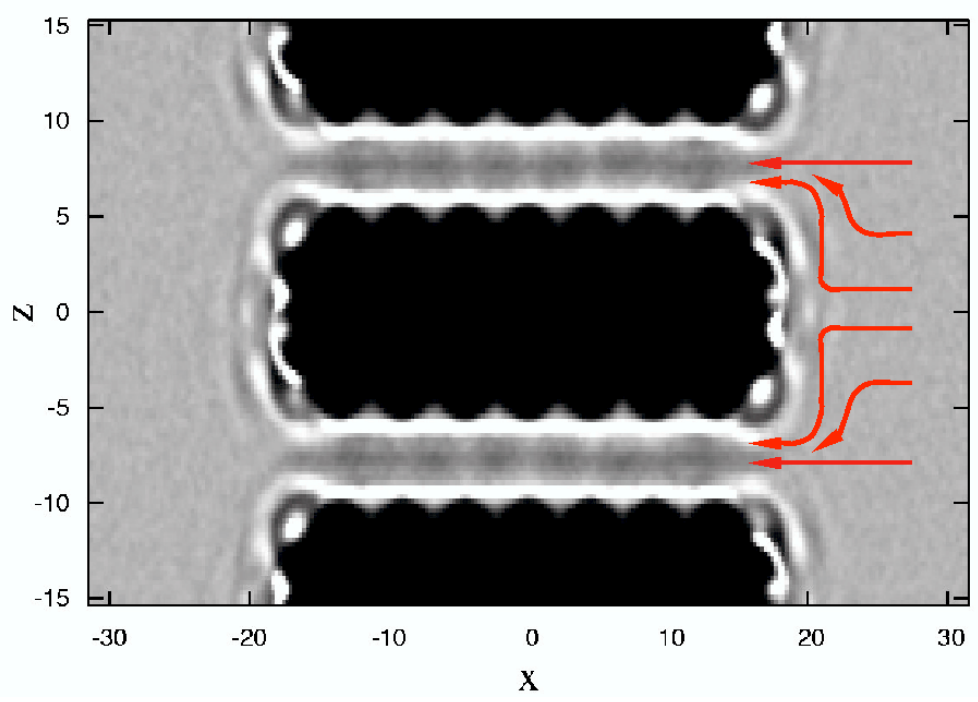


Figure 6

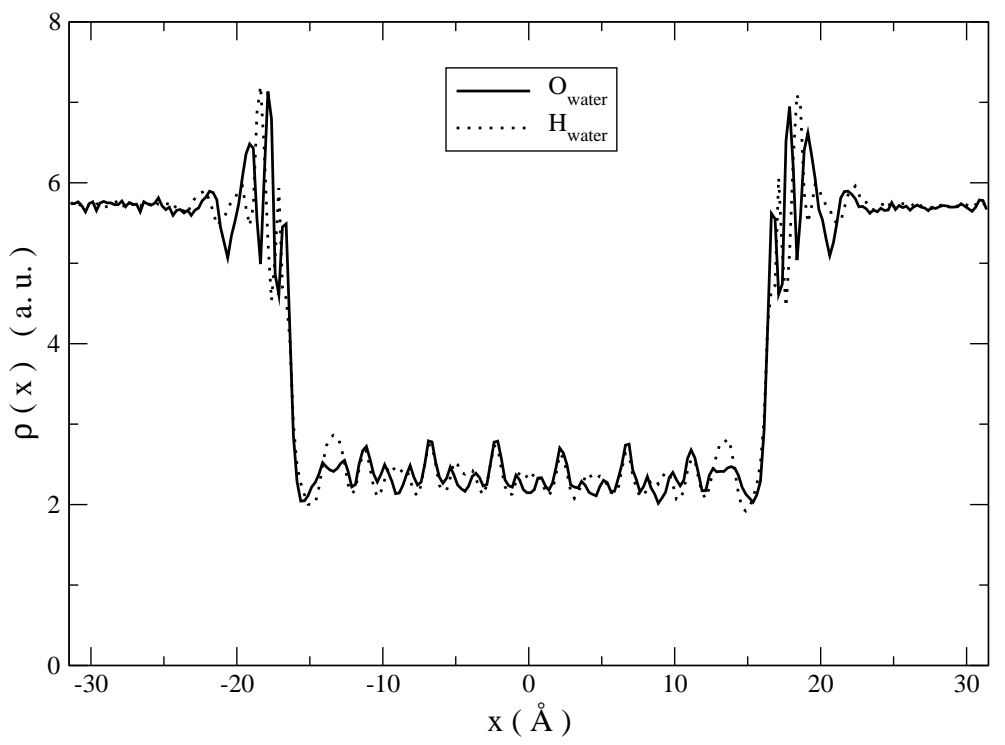


Figure 7

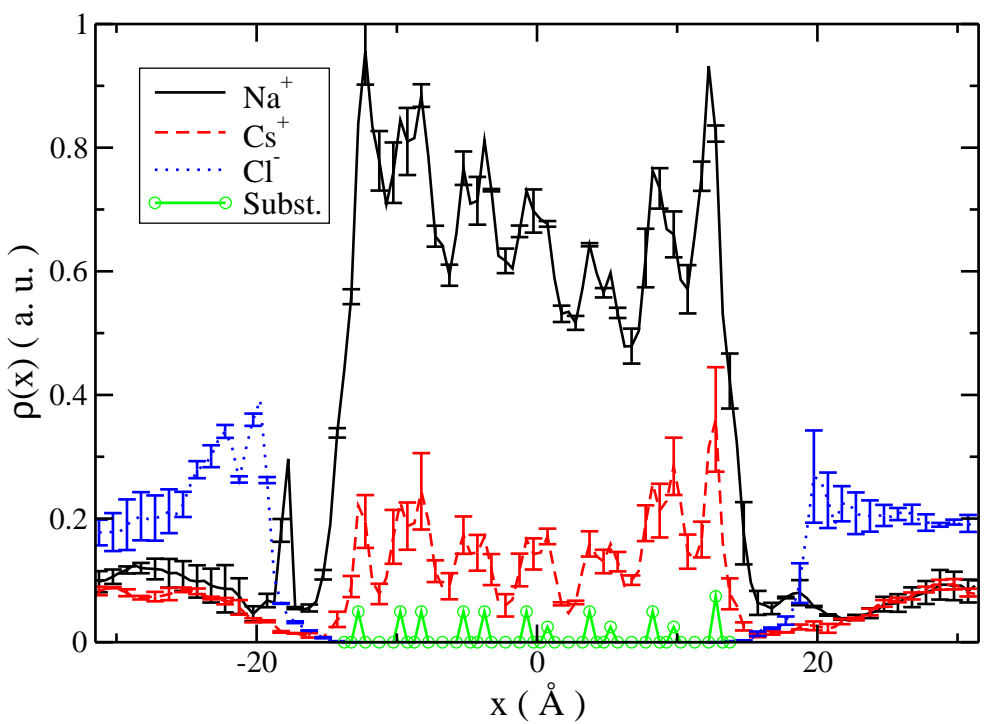


Figure 8
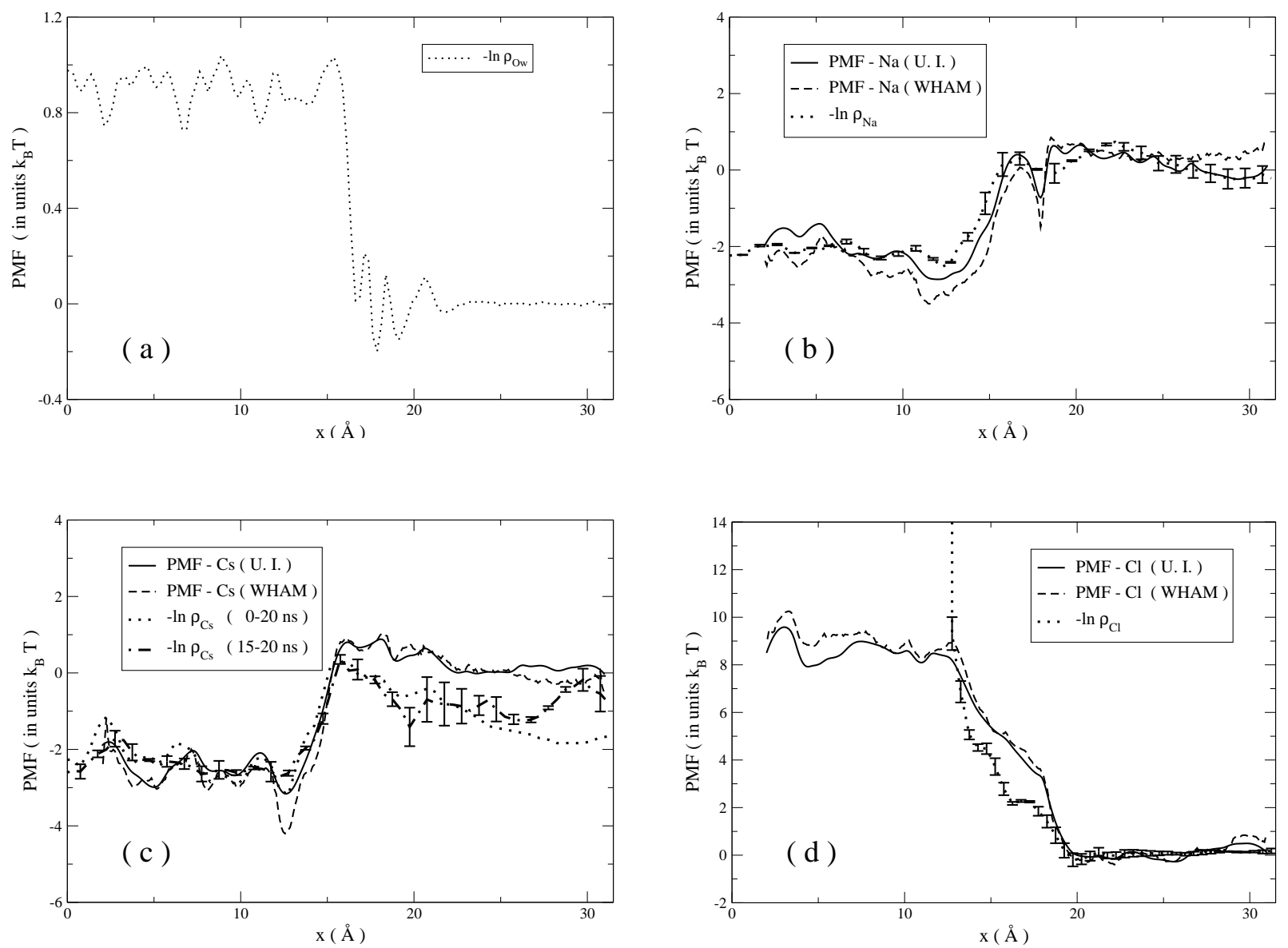
Figure 9

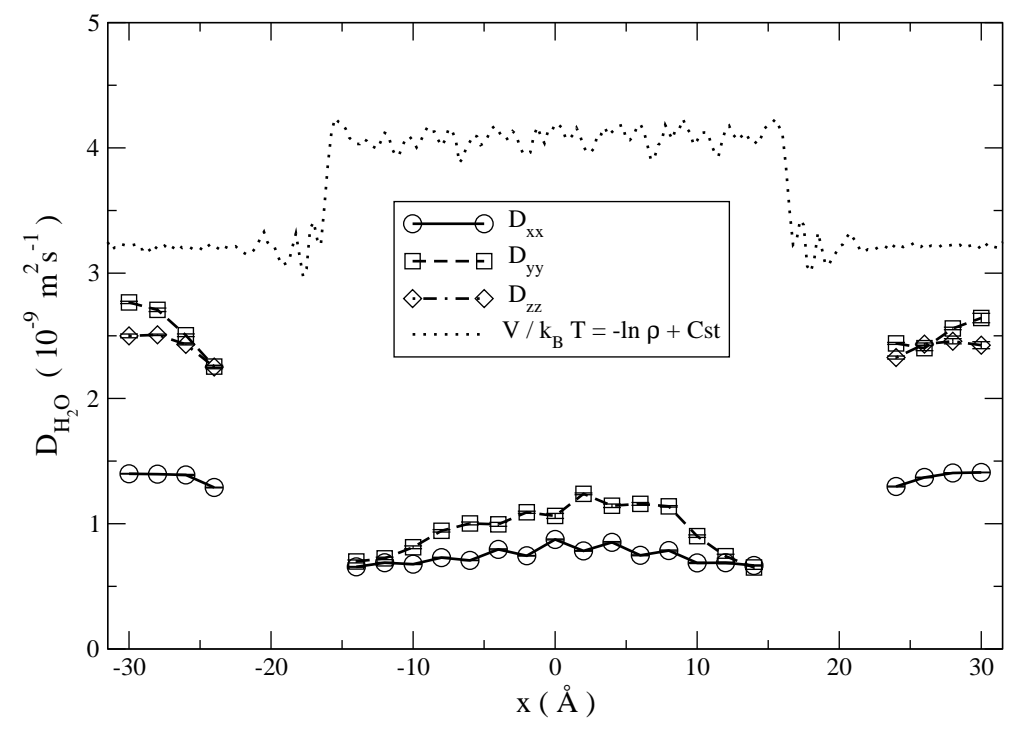

